Horizons philosophiques

\title{
Le journal comme dialogue. Réflexions sur l'écriture de soi et l'authenticité à partir des Carnets de la drôle de guerre de Sartre
}

\section{René Bolduc}

Volume 10, numéro 1, automne 1999

Écritures et confessions

URI : https://id.erudit.org/iderudit/801115ar

DOI : https://doi.org/10.7202/801115ar

Aller au sommaire du numéro

\section{Éditeur(s)}

Collège Édouard-Montpetit

\section{ISSN}

1181-9227 (imprimé)

1920-2954 (numérique)

Découvrir la revue

Citer cet article

Bolduc, R. (1999). Le journal comme dialogue. Réflexions sur l'écriture de soi et l'authenticité à partir des Carnets de la drôle de guerre de Sartre. Horizons philosophiques, 10(1), 155-165. https://doi.org/10.7202/801115ar d'utilisation que vous pouvez consulter en ligne. 


\section{LE JOURNAL COMME DIALOGUE. RÉFLEXIONS SUR L'ÉCRITURE DE SOI ET L'AUTHENTICITÉ À PARTIR DES CARNETS DE LA DRÔLE DE GUERRE DE SARTRE.}

Soi se cherche, se creuse, s'espionne, se viole. II veut se surprendre, mais surprendre quoi ou qui au juste? Sartre dirait qu'il ne s'agit sûrement pas de trouver un soi figé, une substance fixe car ce serait courir le risque de faire de soi une chose définitive, une pâte dure, ininformable puisque déjà formée. Comment éviter alors d'être dupe d'un soi objectivé, d'un pour-soi devenu en-soi?

Par ailleurs, s'écrire, être à soi-même son propre champ d'investigation, se croire le dépositaire de la seule et unique vision du monde possible, c'est-à-dire la sienne, est-ce s'isoler dans une sorte d'ego transcendantal désincarné, délié du monde et des autres?

Répéter le destin de Narcisse cherchant désespérément son image, c'est inaugurer une sorte de jeu de miroir où la conscience se reflète à l'infini. Difficile de s'y retrouver dans ce dédale d'identités. Où rencontre-t-on, en effet, le soi authentique? On emprunte alors un masque, on devient une personne, on «joue» à être soi et on reste lucide de ce jeu : «\|l n'est pas possible de saisir soi-même comme conscience sans penser que la vie est un jeu", écrivait Sartre dans Les carnets de la drôle de guerre (396), reprenant à son compte une phrase de Schiller. ${ }^{1}$ Cela entraîne un conflit mettant en scène au moins deux forces opposées : d'un côté, l'appel identitaire auquel il est vain de résister; il exige constamment de sculpter une certaine forme de présence authentique à soi, c'est-à-dire être enfin quelqu'un, une personne; de l'autre, une conscience malheureuse, nostalgique d'une innocence perdue; elle souffre de ne point pouvoir coïncider parfaitement avec elle-même. La conscience, disait Nietzsche, a perdu sa légèreté instinctive; elle est devenue un poids lourd à porter.

Ainsi, avant même d'entrer en relation avec l'autre, l'existence est

1. "L'homme n'est pleinement homme que lorsqu'il joue." (Schiller). La pagination entre parenthèses est celle de Les carnets de la drôle de guerre. Novembre 1939-Mars 1940 parus aux éditions Gallimard en 1983. 
conflit; déjà une forme de dialogue avec soi-même s'élabore entre l'idéal et la réalité du soi, entre ses possibilités et sa facticité. Telle se présente la condition humaine : le pour-soi, pour être authentique, ne saurait se réduire à son entière immédiateté. II est projet inachevé, ayant toujours à-venir. Se satisfaire de son être comme d'un en-soi relève de la mauvaise foi. Cette attitude réifiante de linauthenticité peut prendre alors différentes formes : le déterminisme, l'esprit de sérieux ou même la sincérité exacerbée. Toutes ces formes peuvent devenir des traces écrites.

"Le passé n'est autre chose que l'existence du pour-soi sur le mode de l'en-soin. (262)

S'écrire, procéder à une sorte de strip-tease de l'âme, correspondre avec soi-même, c'est courir ce beau risque d'empâter sa liberté dans la matière des mots. Assumer cette aventure de la liberté c'est risquer de n'avoir été que ce qu'on a écrit soi-même sur soi. S'écrire, c'est hâter, en quelque sorte, la chute de son présent vers un passé réifié. C'est jeter derrière soi des possibilités du haut d'un présent toujours fuyant. Soidevenant laisse derrière lui des traces de soi-devenu. Le passé «a donc le double caractère d'être un pour-soi immobilisé, figé, un pour-soi devenu chose, c'est-à-dire un événement pétrifié et un ayant-eu-avenir, que cet avenir ait été ou non réalisé" (277). Voilà le risque de cette plongée dans un ayant-été.

Sous l'emprise de la mauvaise foi, quand on voudra se peindre (ce "sot projet" reprochait Pascal à un Montaigne pourtant pas si égocentrique que ça), on pourra tomber dans l'artifice de l'auto-représentation complaisante ; certains se regarderont ou se liront écrire comme d'autres s'écouteront parler. II ne suffira pas d'écrire sur soi pour que la conscience puisse prétendre à une certaine forme d'authenticité. Succombant à l'illusion biographique, la conscience se délectera de cet état originel impossible duquel elle se croyait déchue : enfin être totalement ce qu'elle est et ne plus être ce qu'elle n'est pas. Puis, constatant l'imposture, elle s'arrachera de nouveau à elle-même. La conscience est constamment tiraillée entre ces deux désespoirs : désespérer de ce que nous sommes et désespérer de ce que nous ne sommes pas encore.

"ll est beaucoup plus facile de vivre propre et authentique dans la guerre que dans la paix." (242) 
Durant la «drôle de guerre», plus particulièrement de septembre 1939 à juin 1940, Sartre est très productif. Tout en travaillant intensément à sa trilogie des Chemins de la liberté et en entretenant sa volumineuse correspondance, il remplit à pleine capacité quinze cahiers de journal dont seulement cinq, les autres ayant été perdus, deviendront Les carnets de la drôle de guerre. À cette époque, l'auteur de la Nausée sera affecté au service météorologique : il envoie des ballons pour contrôler la direction du vent. Ainsi en va-t-il de ses carnets : il procédera au relevé des différentes facettes de ses pensées. II a amplement le temps de lire et, surtout, d'écrire. Cela durera neuf mois. Une vraie «drôle de guerre».

Pour l'existentialiste s'ouvre une brèche dans le temps : une période à la fois artificielle et propice commence. Jeté dans cette situation forcée il va écrire parfois jusqu'à douze heures par jour, sans arrêt, comme quelqu'un qui a besoin d'oxygène, comme quelqu'un qui veut fuir le monde, conserver son territoire et se libérer de ce qui le contrarie. ${ }^{2}$ Par l'écriture, Sartre retrouvera cet espace vital que la situation historique lui avait dérobé. Même s'il admit à Paulhan hair le genre autobiographique, le qualifiant de "mesure d'hygiène», il avait déjà avoué à Simone le Castor que ce carnet avait au moins le mérite de faire naître ses idées. ${ }^{3}$ En effet, on y retrouve beaucoup de croquis et de développements assez libres d'idées qu'on retrouvera plus tard dans L'être et le néant. Cependant il ne s'agira pas non plus de notes prises exclusivement en vue de ses œuvres subséquentes. Constitués de remarques entremêlées sur la "drôle de guerre" dans laquelle la France se trouve plongée, ses carnets sont aussi l'occasion de s'exprimer sur l'authenticité, ses liens forcés avec ses compagnons de fortune, sur ses livres parus ou à paraître, ses lectures et commentaires de lecture, incluant ses réactions par rapport à d'autres journaux : Gide, Green, Dabit, Renard, France, Goncourt. On y retrouve même un poème donné «par mortification".

" Je me pare sans cesse car je me décris sans cesse (Montaigne)".

C'est la première fois (et bien avant Les mots de 1964) que Sartre se contraint à retourner littéralement sur soi. On sait que Sartre a poli presque

2. Cf. Annie Cohen-Solal, Sartre. 1905-1980, Paris : Gallimard, 1985, p. 199 et ss.

3. Ibid., p. 200. 
à outrance cette œuvre qu'est l'autobiographie de sa jeunesse. ${ }^{4}$ Que représentent Les mots par rapport aux Carnets? Où est le "vrai» Sartre, pourrait-on demander? Dans la saisie instantanée du soi (ou du soi instantané) que ces carnets nous livrent? Sartre est dans toutes ses œuvres et au delà, cela va sans dire. La question peut paraître oiseuse. Comme si on demandait à une assemblée de "soi-même" : que le vrai soi se lève! Sûrement que tous ces soi, la réalité humaine étant multiple, se lèveraient d'un seul bond, autant ceux que Sartre désigne comme étant de mauvaise foi que ceux se proclamant les champions de la sincérité; autant les aliénés, les "quotidiens", que les authentiques. Où est le vrai soi? En ce qui concerne Sartre, si on réduit la question à la production écrite, la réponse pourrait être : dans ses romans, son théâtre, ses nombreux essais politiques, littéraires, philosophiques, etc. Tout cela révèle la personnalité de cet auteur prolifique. Quand la personnalité occupe l'avant-scène, il y a alors la correspondance. Les entretiens. Les autobiographies. L'autobiographie brute du carnet plus porté sur le présent et les pensées saisies au vol et l'autobiographie plus élaborée, "œuvrée», de mémoires reconstruisant le passé.

Les écrits autobiographiques ne constituent pas de bloc monolithique : il y a plusieurs genres : mémoires, journaux intimes, carnets, confession. On n'a qu'à comparer le narrateur des Mots et celui des Carnets, pourtant deux genres autobiographiques, pour mesurer l'étendue de ces différences. En fait, le narrateur des Mots est plus près de celui du roman La Nausée. ${ }^{5}$ Tous ces genres ne sauraient pas non plus être qualifiés d'œuvres proprement dites.

Établissons deux extrêmes du genre autobiographique à partir de leur mode de production : cela va des notices quotidiennes plus ou moins reliées entre elles (par exemple, des carnets) au journal longuement mûri qui tente une synthèse parfois expiatoire, parfois justificatrice (par exemple, des mémoires, des confidences). Cela va aussi de l'obsession égotiste plutôt étroite à la tentative de mieux se comprendre en tenant compte de sa

4. Le manuscrit des Mots a été uretaillé, remâché, retravaillé, avait fait l'objet de pages et de pages de brouillons, de pages et de pages de ratures, de reprises, d'ourlets, de pièces, de soudures et de corydrane, dans un patient travail d'artisan qu'il n'avait jamais auparavant, qu'il n'aura jamais plus tard, consacré à aucune autre de ses œuvres". Ibid., p. 566 . II l'avouera à Simone de Beauvoir dans cet entretien autobiographique qu'est la Cérémonie des adieux.

5. II ne faudrait pas pour autant conclure qu'll y a un "vrain Sartre qui écrit sur lui-même et un Sartre moins "vrai» qui écrit des romans. Il admit même à Michel Contat qu'll avait atteint sa vénité autant à travers Roquentin ou Mathieu, personnages des romans La Nausée et des Chemins de la liberté, qu'à travers Les Mots. C.f. "Entretiens sur moi-même", in, Situations, X, p. 143. 
situation historique et de la compagnie des autres qui comptent. S'il est difficile d'établir la démarcation entre un journal qui devient une œuvre, voire un chef d'œuvre, et un journal auquel on ne peut attribuer cette qualité, sans quoi la notion d'œurre perdrait tout son sens, on peut quand même faire quelques distinctions. Sans doute faut-il d'abord que la littérature intimiste veuille chercher public, ce qui est loin d'être le cas de tous les journaux. Ensuite, se complaire uniquement dans l'intimité pure (mais est-ce vraiment possible?) peut confiner rapidement à l'insignifiance. C'est une banalité de le rappeler, mais ce n'est pas parce qu'il s'agit de moi pour que, de facto, cela devienne intéressant pour tous. Mais sans nous perdre dans le labyrinthe axiologique, disons qu'on peut difficilement qualifier d'œuvre l'autobiographie du type carnet et ce, malgré toutes les qualités littéraires qu'elle puisse offrir. Darwin n'a pas publié ses carnets de voyage pour présenter sa fameuse théorie mais bien L'origine des espèces. II y va de l'être même du carnet qu'il ne soit pas œuvre. La distinction que je fais ici, je le rappelle, ne concerne non pas tant les résultats obtenus que le mode de production de l'écrit autobiographique. Par exemple, le carnet sera beaucoup plus axé sur un présent immédiat, à chaud si on veut, tandis que des mémoires synthétisantes mettront en perspective un passé plus ou moins proche. Ces dernières tendront vers quelque chose d'achevé, se suffisant à lui-même quoique toujours ouvert à l'interprétation, tandis que les carnets tendront vers quelque chose de plus instrumental, de furtif et de gratuit. «Entière gratuité de ce carnet (...) Si j'écrivais un livre composé, j'irais plus loin, à la manière des soldats en guerre que l'on contraint toujours de tenir un peu plus qu'ils ne peuvent." (279) Les mémoires sont construites et un certain sens global est donné à cette construction dont on se permettra parfois d'en modifier le sens. Un carnet sera relu pour en retirer des informations mais il ne sera pas "construit". II semblerait absurde aussi de "corriger" ses anciennes impressions personnelles. Cela semble d'autant plus absurde que destinateur et destinataire se confondent ici. Mais de la même façon que des états totalitaires font disparaître de leurs archives des pans entiers de leur histoire, il peut arriver que soi puisse vouloir exercer sa domination sur ses traces passées avec lesquelles il n'est plus d'accord. Le soi, "cette tour où l'on monte et où on se regarde" (Sartre), succède historiquement à d'autres formes de soi; autre temps, autres tentations de domination. On dira que ce dessaisissement de son passé est un acte libre, qu'on ne peut pas, de toute façon, se révéler entièrement, que la liberté est à la fois affirmation et négation. N'empêche, 
à cause de cette dictature du présent une possibilité de dialogue avec un soi de jadis voit ainsi ses chances s'amoindrir.

"La conscience de soi n'existe comme moi que si elle se voit dans une autre conscience de soi (Hegel)".

Ce qu'on exige d'un témoignage, c'est qu'il soit authentique, peu importe qu'on ait affaire à un carnet, sur lequel on ne reviendra plus, sinon pour le consulter, ou à un journal habilement "œuvré" qui a pu puiser à même des carnets. Le soi du carnet n'est pas d'emblée plus authentique parce que supposément plus spontané, moins censuré. Non. Celui-ci est tout aussi capable de déguisement que la plus habile des réflexions. On croyait s'écrire tel quel mais ce n'était pas encore ça. Dans un entretien avec Michel Contat, Sartre avouera qu'il est difficile de nous donner jusqu'au bout parce que nous nous connaissons peu. ${ }^{6}$ Plus que jamais il faut éviter de tomber dans "lillusion biographique, qui consiste à croire qu'une vie vécue peut ressembler à une vie racontée." (105-106)

Sartre, ardent défenseur de l'authenticité, procédera à quelque autoviolation de la conscience. II s'efforcera de se mettre à jour. Comment éviter alors le piège de la complaisance? Comment éviter surtout le piège de lillusion de l'accès immédiat et exclusif à soi? Comment rester fidèle à soi, avec et malgré les mots? À moins que l'écrit sur soi ne soit déjà beaucoup plus qu'un simple monologue. II est communication avec soi, certes, qui risque de devenir communication avec l'autre, en devenant public, mais le soi n'est peut-être pas aussi seul qu'il croit l'être au moment même où il s'écrit. Soi n'est pas tant un autre qu'un étant-avec-les-autres. "Et ces carnets, au fond, sont une manière de vivre public encore" (331), reconnaît Sartre. Dans son désir de «translucidité totale», il n'entend pas, et ne saurait de toute façon, couper les liens avec les autres. L'être humain, en tant qu'être dialogique bâtit son identité en communication avec et contre autrui. Si la quête de l'identité ne se conçoit que parce qu'il y a différence avec l'autre, cette différence lui devient alors essentielle.

Que vaut alors cette exigence de connaissance de soi via le journal si l'autre me parasite constamment en dépit de moi-même? Si Je, en définitive, est désespérément un autre? L'exercice n'est pas pour autant vain. II faut le remettre à sa juste perspective. C'est ce que propose Francis Jacques dans l'esprit des penseurs dialogiques:

6. Ibid, p. 144-5. 
Est-ce à dire que l'homme ne puisse se connaître? On n'ira pas jusque là. Mais la connaissance de soi ne procède pas d'un dédoublement réflexif. Voilà le point. Sans doute le moi n'est-il pas fait pour se voir ou se dire lui-même. (...) Mon affaire est de me manifester. Non certes une auto-manifestation : I'homme n'apprend à se connaître que par le long détour de l'interprétation des signes qu'il émet en situation communicative. La compréhension de moi-même est toujours médiate. Je ne me comprends qu'à travers signes et œuvres, produits en relation avec les autres. Là est mon miroir réel. ${ }^{7}$

Acceptons d'emblée le caractère foncièrement dialogique de l'être humain. Même à notre insu, les écrits les plus intimistes recèlent alors ce caractère : je ne suis moi que parce qu'il y a toi et les autres. Perdre de vue cette dimension communicative peut avoir des conséquences qui atteignent à la morbidité. N'être que pour soi ce sera, dans certains cas, mourir un peu :

Un café chaud, une cigarette, une chaise, une table, une fenêtre. Du papier, un stylo. Le reste est sans importance, ne peut rien modifier à ce qui se passe alors chez moi. Nudité intérieure. Le sentiment de n'exister que par soi-même, ce qui est d'ailleurs la vérité si on se donne la peine d'y réfléchir un tant soit peu. Aucune filiation qui tienne, pas de territoire autre que celui de sa propre ossature, de ses propres mots. Ici la mort est la seule liberté, le seul acte qui dépende entièrement de nous. 8

Peut-être vaut-il mieux alors, conscient des limites d'être le propre auteur de son journal écrit ou vécu, se perdre comme des Gauguin, Van Gogh et Rimbaud pour mieux se retrouver? Égarer sa trace pour mieux la ressaisir, quitter la familiarité de son terroir pour mieux l'apprécier? Sartre avouera dans ses carnets son net complexe d'infériorité vis-à-vis de ces défricheurs puisqu'ils ont su aller jusqu'au bout d'eux-mêmes. Être authentique, pour eux, c'était se perdre. II admire même davantage Rimbaud puisqu'il a renoncé à l'écriture. Pour Sartre cela représenterait une véritable perte existentielle. Une véritable révolution eût été, dans son cas, non pas, par exemple, de renoncer au Nobel, mais de renoncer à écrire. Sartre appelle "craquements existentiels" cette révolution par laquelle émerge un soi plus véridique: "Je pense de plus en plus que, pour atteindre l'authenticité, il faut que quelque chose craque". (43) Ne pouvant s'administrer sa propre médecine, Sartre ne renoncera pas à l'écriture : "Je me suis préservé contre les craquements. Je suis ligoté à mon désir d'écrire.» (43)

7. Francis Jacques, Différence et subjectivité, Paris : Aubier/Montaigne, 1982, p. 204.

8. Jean-Pierre Guay, Journal, tome 1, Montréal, CLF, 1986, p. 34; cité par Marc Chabot, Le joumal des autres, Montréal, éd. St-Martin, 1988, p. 184. 
Autant demander à un poisson de vivre hors de l'eau! "Je vois bien que je rêve seulement de remettre en question mon désir d'écrire parce que si vraiment j'essayais, fût-ce une heure, de le tenir en suspens, de le mettre entre parenthèses, toute raison s'écroulerait de remettre quoi que ce soit en question." (44) Ne plus écrire, surtout pour le Sartre mobilisé, signifierait en même temps ne plus être soi. II n'y a pas d'autre authenticité pour lui que celle obtenue par le matériau littéraire. II a besoin des mots pour maîtriser sa vie, les mots d'une conscience s'épiant elle-même, toujours à l'affût de soi, dans cet engluement de la drôle de guerre.

Sartre n'élève pas de temple dédié au culte de sa propre personnalité. Les descriptions de son existence, même la plus intime, sont le plus souvent encadrées de réflexions plus fondamentales. Elles s'immunisent ainsi contre l'insignifiance. Mais à force de se soumettre ainsi à l'autoanalyse existentielle, et malgré toute la bonne foi de la lucidité, il arrive que le dédoublement réflexif nous désolidarise du présent. "Dresser l'inventaire perpétuel de ce qu'on est, c'est se renier constamment et se réfugier dans une sphère où l'on n'est plus rien, qu'un pur et libre regard ${ }^{9}$ ". En jetant le filet des mots sur soi pour se ressaisir, il peut paraître difficile de parler d'authenticité. Si les mots accusent un décalage littéraire sur le réel, nous serons à la fois dans le coup et nous n'y serons plus parce que nous nous y verrons dedans :

C'est vrai, je ne suis pas authentique. Tout ce que je sens, avant même que de le sentir je sais que je le sens. Et je ne le sens plus qu'à moitié, alors, tout occupé à le définir et à le penser. Mes plus grandes passions ne sont que des mouvements de nerfs. Le reste du temps, je sens à la hâte et puis je développe en mots, je presse un peu par ici, je force un peu par là et voilà construite une sensation exemplaire, bonne à insérer dans un livre relié. (82)

Oui, les mots nous pétrissent la réalité. Ils créent une sorte de décalage. Mais sans les mots, la réalité humaine équivaut à une contradiction dans les termes. "Le langage est la maison de l'être", disait Heidegger. Le langage me sortant de moi-même, me mettant, pour ainsi dire, au

9. Sartre, L'être et le néant, Paris : Gallimard (Tel), 1943, p. 102. II est vrai que toujours penser à soi est aussi absurde que de vouloir toujours penser respirer pour ne pas trépasser. Cela peut même devenir obsédant, voire schizoìde, pour qui souffre d'insécurité ontologique, dira l'anti-psychiatre Laing, lui-même influencé par les analyses sartriennes de l'existence. Dans Le moi divisé, il rapporte un cas pathologique : «Lorsque je me suis soudain rendu compte que je ne pensais pas à moi, j'ai éprouvé une peur affreuse et un sentiment d'irréalité. Je ne dois jamais m'oublier, fût-ce pendant une minute.» 
monde est à la fois obstacle et condition de mon authenticité puisque "c'est bien moi, ce redoublement continuel et réflexif, cette précipitation avide à tirer parti de moi-même, ce regard". (82-83) "Je ne suis qu'orgueil et lucidité». (83) Cette anti-œuvre que sont les carnets n'échappe pas, malgré sa gratuité, à une donation globale de sens. lls sont peut-être médiocres, d'après l'aveu même de l'auteur, mais ils ne sont pas pour autant insignifiants. Pour Sartre, qui n'est ni honteux ni fier de ses pensées, il ne s'agit pas de faire un livre de confessions et d'auto-légitimation à la Rousseau ou d'expiation aux accents religieux à la Gide, mais de livrer témoignage, pour les autres - «le témoignage d'un bourgeois de 1939 mobilisé» (90) — ou de tirer au net, pour lui-même. Alors peu importe qu'il ait de la valeur ou non, cet humble témoignage se distinguerait dans sa non-distinction; histoire d'une personne qui n'est personne, d'une personne dont l'existence est semblable à celle de millions d'autres personnes, d'une personne qui s'est trompée comme des millions d'autres avant elle se sont trompées. Ainsi la singularité rejoint-elle l'universalité. La faute de voyeurisme narcissique et excessif se voit ainsi absoute par avance. Dire Je, c'est déjà rejoindre l'universalité des Je, c'est baigner dans un langage dont je n'ai pas établi moi-même les règles. Je n'est pas seulement un autre, je est tous les autres. À ce compte, uil n'en faut pas plus pour me convaincre que tout ce que j'écris est intéressant..." (91).

Est-ce que Sartre voulait dire que tous les témoignages se valent en autant qu'ils soient humains? Que le sien est désespérément pareil à tous les autres? Peut-il faire fi de tout sentiment d'élection, de témoignage exemplaire ? Pourquoi écrire si on se réduit constamment à n'être rien? Sartre a retenu une leçon de Gide : il s'agirait, dans le dédoublement réflexif, d'une ruse de la raison devant nous convaincre, malgré tout et malgré soi, d'écrire. Cette ruse excuserait par avance nos imperfections. Elle nous rappelle notre finitude, notre conditio humana, et nous convainc de poursuivre malgré tout. Ainsi, à défaut d'être une révélation de grandeur (90), la production d'un journal peut-être médiocre serait moralement cautionnée comme une représentation de tous les médiocres, les inauthentiques ayant droit, eux aussi, à leur être historique.

Mais est-ce vraiment comme un humble parmi les humbles que l'auteur des Chemins de la liberté se considérait? Sartre, se projetant dans l'avenir, se faisait d'emblée appartenir aux grands. Ainsi projetait-il rêveusement sa future vie de grand écrivain. Cette esthétisation de l'existence 
accentuait une sorte de mélancolie, une impression d'irréalité, une nostalgie de ce qui n'a point encore été. Le soi projeté et idéalisé rejette le soi que désespérément nous sommes et que nous ne voudrions pas être. "En somme j'aurais voulu être sûr de devenir un grand homme plus tard, pour pouvoir vivre ma jeunesse comme une jeunesse de grand homme." (97) Sartre tombe pendant un certain temps dans l'illusion biographique : il envisage "chaque moment présent du point de vue d'une vie faite, pour être exact il faudrait dire, du point de vue d'une biographie." (103) "Devenir le spectateur de sa propre vie permet d'échapper aux souffrances de la vie», écrivait Wilde. Vivre le livre dont on est à la fois l'auteur, le narrateur, le personnage principal et l'unique lecteur ! Attitude de mauvaise foi. Effet d'une pensée essentialiste. Faire semblant d'être ce qu'on n'est pas encore. S'engloutir dans le grand fantasme de lillusion rétrospective. À quoi sert de jouer la pièce si de toute façon elle est écrite d'avance ? Au moment même où l'on ne voudrait pas être dupe, où l'on voudrait récupérer sa liberté en coupant tous les liens, on se laisse naturellement séduire par une beauté dont on définit soi-même les règles. Mais suis-je pour moi-même mon meilleur miroir? "Quel œil peut se voir lui-même? ${ }^{10 " . ~ " J ' e s t i m e ~ q u e ~}$ toute cette région sombre que nous avons en nous-mêmes, à la fois sombre pour nous et sombre pour les autres, nous ne pouvons l'éclaircir pour nous-mêmes qu'en essayant d'être clairs pour les autres ${ }^{11}$ ".

Sartre a cru un certain temps que l'authenticité ne s'acquerrait que dans l'esseulement. "Je crois que j'étais authentique avant ma permission. Sans doute parce que j'étais seul.» (267) Mais à quelle sorte d'authenticité pourrais-je bien prétendre si je me persuade que les relations avec les autres me dépouillent de mon être? À quelle sorte d'identité puis-je aspirer si je coupe les liens avec ma situation historique, c'est-à-dire avec notre être-en-commun? Dans un écrit posthume de Sartre on peut lire :

S'il y a un mode d'être commun qui est l'inauthenticité, alors toute l'Histoire est inauthentique et l'action dans l'Histoire entraîne à l'inauthenticité ; l'authenticité retourne à l'individualisme. Réciproquement, si la nature de l'homme est au bout de l'Histoire, l'inauthenticité doit être voulue pour elle-même comme la condition même de la lutte historique. 12

10. F. Jacques, op.cit., p. 196.

11. Sartre, «Entretiens sur moi-mêmen, in, Situations, X, op. cit., p. 143.

12. Sartre, Vérité et existence, Paris : Gallimard 1989 (1948), p. 11 
Obnubilé par son rapport à l'écrit en général et par son désir d'authenticité en particulier, Sartre vit son an-historicisme même pendant sa mobilisation, événement pourtant historiquement significatif s'il en est un. Déjà, lors de son séjour à Berlin en 1933-34, soit peu après la prise du pouvoir par Hitler, Sartre, imprégné de phénoménologie husserlienne, met le monde politique entre parenthèses; c'est à peine s'il prend note des bouleversements historiques. Le 6 mars 1940, il écrit dans ses carnets : "Je ne suis solidaire de rien, pas même de moi-même; je n'ai besoin de personne ni de rien." Plus loin : «N'ayant pas de grande passion sociale, vivant en dehors de ma classe et de mon temps, je ressemble au lapin de Claude Bernard, isolé aux fins d'expériences, à jeun et qui se digérait lui-même."(356) On l'a déjà noté, Sartre se protège de la réalité en la faisant passer par la moulinette de l'écriture. "Tout ce que je sentais, aussitôt je le saisissais avec des gants, je l'exprimais en mots avant même de l'avoir laissé atteindre son plein développement (...)" (330). II n'est pas particulièrement fier de cette image de distanciation. En fait il veut changer et un changement s'opérera justement lors de sa captivité à Trèves où il fera une expérience, disons, plus positive de l'être-en-commun.

L'écriture de soi, comme n'importe quel autre écrit, n'épuise jamais tout à fait son sens, ni pour l'auteur, ni pour le lecteur. Si elle est dialogue avec soi c'est parce que nous sommes avec les autres. En fait, on ne se connaîtra véritablement mieux soi-même qu'en situation de communication. Ceci ne veut pas nécessairement dire qu'il faille factuellement être deux; comme êtres dialogiques, nous le sommes toujours déjà. Plutôt qu'une coupure radicale avec l'autre, il faut considérer l'entretien avec soi-même comme une modification de notre mode ordinaire d'être avec autrui. Même si l'autobiographie se laisse guider par la complaisance ou l'amour-propre, il ne faut pas voir par là une attitude de détachement par rapport au monde; au contraire, ces attitudes montrent que nous sommes dépendants de ces passions sociales. II ne s'agit pas non plus seulement d'une dépendance psychologique, d'un trait de caractère modifiable à souhait par une conscience souveraine, mais d'une dépendance ontologique, irréductible, par laquelle nous trouvons notre authenticité.

René Bolduc

Québec 